\title{
THREE-DIMENSIONAL DISTRIBUTION OF THE NUCLEAR MIRRORS IN NGC 1068
}

\author{
MAKOTO KISHIMOTO \\ Kyoto University \\ Sakyo-ku, Kyoto 606-01, Japan
}

\section{Introduction}

Recently, the HST imaging observations have revealed that the central arc second region in NGC 1068 has a clumpy structure (Macchetto et al., 1994) and the HST UV imaging polarimetry has revealed the centrosymmetric pattern of the position angles of polarization (Capetti et al., 1995), which indicates that the medium in this region is actually scattering the radiation from a small source, in accordance with the unified model of active galactic nuclei (Antonucci \& Miller, 1985).

We use those UV imaging polarimetry data, to extract the polarization of each clump and determine each scattering angle (or "viewing angle" of each clump), based on certain assumptions described below. This leads us to know the three-dimensional distribution of the scattering clouds, with respect to the location of the central radiation source.

\section{Necessary Assumptions and Method}

We assume; (1) The nuclear scattering gas ( $100 \mathrm{pc}$ scale $)$ consists of several clumps (each clump size is less than $\sim 10 \mathrm{pc}$ ), as indicated by the HST images; (2) Those clumps are being illuminated by single, small source of radiation; (3) Only the scattering process is the cause of the polarization; (4) All of the UV continuum light $(\sim 2500 \AA)$ is the scattered radiation, based on the results from Antonucci et al. (1994) and Tran (1995).

Under those assumptions, "viewing angle" of each clump can be determined from its polarization. Two viewing angles, however, are derived from single value of polarization; the forward and backward-scattering case. This converts to the two positions along one line of sight. 

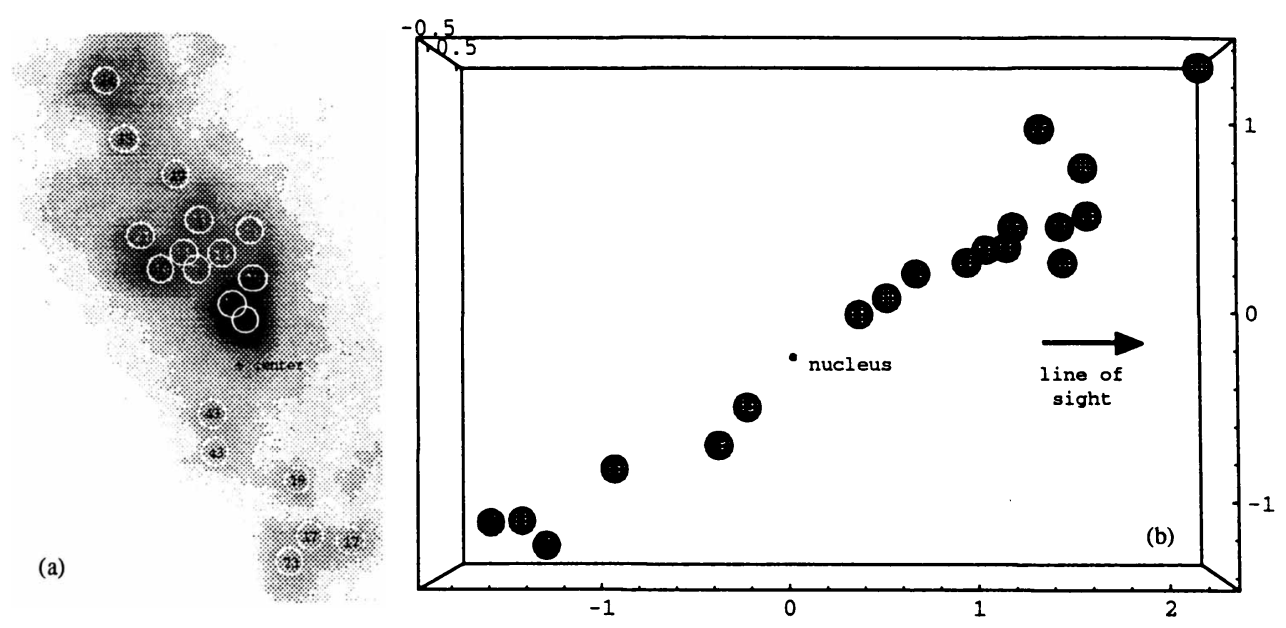

Figure 1. (a) Polarization of each clump. The center of centrosymmetric pattern of PA's is also indicated. The scale is $\sim 2^{\prime \prime} \times 3^{\prime \prime}$. North is up, east is left. (b) The inferred view of the same region as (a), but viewed from east side. The line of sight is from the right.

\section{3D Mapping}

¿From the archival HST/FOC data, we extracted the polarization of each clump (Fig 1a), from which the viewing angles of each clump were calculated and the three-dimensional locations with respect to the hidden nucleus were determined (Fig.1b). The positions depicted in this figure are only for one of the possible cases. We could select back-scattering or forward-scattering case or even both. We prefer those positions in Fig.1b, however, because there is a evidence that indicates the amount of the interstellar extinction is smaller in the north side of the nucleus than the south and changes rather abruptly between those two regions (Macchetto et al., 1994).

Although ambiguity is large, this result suggests that there is certain linear structure in the nuclear region in this $100 \mathrm{pc}$ scale, which could be related to the jet-like structure in the same scale observed in radio wavelength (Gallimore et al., 1996). For more details, see Kishimoto (1997).

\section{References}

Antonucci, R., \& Miller, J.S. 1985, ApJ, 297, 621

Antonucci, R., Hurt, T., \& Miller, J.S. 1994, ApJ, 430, 210

Capetti, A. et al. 1995, ApJ, 452, L87

Gallimore et al. 1996, ApJ,

Kishimoto, M. 1997, ApSS in press

Macchetto, F. et al. 1994, ApJ, 435, L15

Tran, H.D. 1995, ApJ, 440, 578 